\title{
High COPD prevalence at high altitude: does household air pollution play a role?
}

\author{
Evelyn A. Brakema (1) ${ }^{1}$, Aizhamal Tabyshova ${ }^{2,3}$, Marise J. Kasteleyn ${ }^{1}$, \\ Eveline Molendijk ${ }^{1}$, Rianne M.J.J. van der Kleij ${ }^{1}$, Job F.M. van Boven $\mathbb{1}^{3}$, \\ Berik Emilov², Meerim Akmatalieva², Maamed Mademilov², Mattijs E. Numans ${ }^{1}$, \\ Sian Williams ${ }^{4}$, Talant Sooronbaev ${ }^{2}$ and Niels H. Chavannes ${ }^{1}$ \\ Affiliations: ${ }^{1}$ Dept of Public Health and Primary Care, Leiden University Medical Center, Leiden, The \\ Netherlands. ${ }^{2}$ Pulmonary Dept, National Center of Cardiology and Internal Medicine, Bishkek, Kyrgyzstan. \\ ${ }^{3}$ Dept of General Practice and Elderly Care Medicine, Groningen Research Institute for Asthma and COPD \\ (GRIAC), University Medical Center Groningen, University of Groningen, Groningen, The Netherlands. \\ ${ }^{4}$ International Primary Care Respiratory Group (IPCRG), London, UK.
}

Correspondence: Evelyn A. Brakema, Dept of Public Health and Primary Care, Leiden University Medical Center, Postzone V0-P, Postbus 9600, 2300 RC Leiden, The Netherlands. E-mail: evelynbrakemadagmail.com

@ERSpublications

High indoor PM2.5 levels could explain elevated COPD prevalence in rural, high-altitude settings: prevention is key! http://ow.ly/hvC930mFEKJ

Cite this article as: Brakema EA, Tabyshova A, Kasteleyn MJ, et al. High COPD prevalence at high altitude: does household air pollution play a role? Eur Respir J 2019; 53: 1801193 [https://doi.org/10.1183/ 13993003.01193-2018].

ABSTRACT Studies comparing chronic obstructive pulmonary disease (COPD) prevalence across altitudes report conflicting results. However, household air pollution (HAP), a major COPD risk factor, was mostly not accounted for in previous analyses and never objectively measured. We aimed to compare the prevalence of COPD and its risk factors between low-resource highlands and lowlands, with a particular focus on objectively measured HAP.

We conducted a population-based, observational study in a highland ( $2050 \mathrm{~m}$ above sea level) and a lowland ( $\sim 750 \mathrm{~m}$ above sea level) setting in rural Kyrgyzstan. We performed spirometry in randomly selected households, measured indoor particulate matter with an aerodynamic diameter $<2.5 \mu \mathrm{m}(\mathrm{PM} 2.5)$ and administered a questionnaire on other COPD risk factors. Descriptive statistics and multivariable logistic regressions were used for analyses.

We included 392 participants: 199 highlanders and 193 lowlanders. COPD was more prevalent among highlanders (36.7\% versus 10.4\%; $\mathrm{p}<0.001)$. Their average PM2.5 exposure was also higher (290.0 versus $72.0 \mu \mathrm{g} \cdot \mathrm{m}^{-3} ; \mathrm{p}<0.001$ ). In addition to high PM2.5 exposure (OR 3.174, 95\% CI 1.061-9.493), the altitude setting (OR 3.406, 95\% CI 1.483-7.825), pack-years of smoking (OR 1.037, 95\% CI 1.005-1.070) and age (OR 1.058, 95\% CI 1.037-1.079) also contributed to a higher COPD prevalence among highlanders.

COPD prevalence and HAP were highest in the highlands, and were independently associated. Preventive interventions seem warranted in these low-resource, highland settings. With this study being one of the first spirometry-based prevalence studies in Central Asia, generalisability needs to be assessed.

This article has supplementary material available from erj.ersjournals.com

Received: June 262018 | Accepted after revision: Nov 022018

Copyright $\odot$ ERS 2019. This article is open access and distributed under the terms of the Creative Commons Attribution Non-Commercial Licence 4.0. 


\section{Introduction}

Chronic obstructive pulmonary disease (COPD) is the world's third leading cause of death, accounting for $5.7 \%$ of all deaths globally $[1,2]$. Over $90 \%$ of COPD-related deaths occur in low- and middle-income countries, leading to a substantial clinical, economic and societal burden [3,4]. Gaining insight into risk factors and places where the burden of disease is highest is key to a tailored approach to COPD.

Globally, around 400 million people live at high altitude ( $>1500 \mathrm{~m}$ above sea level) [5], yet it remains equivocal whether COPD prevalence is higher in these settings. Five observational studies in diverse settings reported contradictory findings [6-10]. Additionally, two studies pooled data from various prevalence studies, and while one described a lower COPD prevalence at higher altitude [11], the other observed no difference when adjusting for several risk factors [12]. However, household air pollution (HAP) was not adjusted for in either of these pooled studies. Of note, especially in low-resource settings, HAP is increasingly reported as a major risk factor for COPD [2, 3, 13-18].

HAP is caused by the indoor use of coal and biomass (e.g. animal dung, wood and crop residues), also known as solid fuels. Cooking and heating with solid fuels, as is common in $90 \%$ of rural households worldwide, leads to variably high levels of lung-damaging pollutants. The most exposed are women and their children, as women often prepare the meals above the fire in poorly ventilated houses for several hours daily, keeping their children close to them [13-15]. In 2015, HAP was estimated to be responsible for 4.2 million deaths and 103.1 million lost years of healthy life [19].

Some of the previous studies did report HAP exposure across altitudes, but used self-reported proxies (such as "solid fuel use") $[7,8,10]$. None objectively measured personal particulate matter exposure in vivo, which is needed for accurate pollution assessment [20]. Earlier studies also focused almost exclusively on urban settings, yet rural settings have more limited access to clean fuels and are consequently more severely affected by HAP [14].

Therefore, in this study we aimed to compare the prevalence of COPD and its risk factors between rural, low-resource highlands and lowlands, with a special focus on HAP. We expected that the more extreme climate conditions at high altitude increase the need for (solid fuel-based) heating and for limiting ventilation, thereby increasing the risk of COPD. We therefore hypothesised HAP exposure to be higher at high altitude and, consequently, COPD prevalence to be higher as well.

\section{Methods}

\section{Study design and setting}

We conducted an observational, cross-sectional, population-based study in a lowland and a highland setting in rural Kyrgyzstan. This lower-middle-income country in Central Asia has a population of 5.96 million, with an average life expectancy of 71 years [21]. It has the highest respiratory mortality of all countries in the European Respiratory Society (ERS) "White Book" [22]. Kyrgyzstan offers a large contrast in altitude. Its lowest region (Chui, $\sim 750 \mathrm{~m}$ above sea level) and one of its highest regions (Naryn, $\sim 2050 \mathrm{~m}$ above sea level) were selected as lowland and highland settings. Chui has 756000 rural inhabitants (out of a total of 922000) and hosts the country's capital Bishkek. Its neighbouring region, Naryn, has 245000 rural inhabitants (out of a total of 284000) [23]. We collected data in the lowlands between February 3 and May 7, 2015, and in the highlands between July 16 and September 13, 2014. The colder measurement period in the year in the lowlands compared with the highlands was considered to involve relatively more (solid fuel-based) heating and less ventilation in the lowlands. Hence, seasonal influences would lead to an underestimation of differences in exposure to HAP and in respiratory symptoms.

This study preceded the Free Respiratory Evaluation and Smoke-exposure reduction by primary Health cAre Integrated gRoups (FRESH AIR) project (Netherlands Trial Register identifier NTR5759), an implementation science project targeting chronic lung diseases in low-resource settings [24].

\section{Participants}

Given that no previous studies of COPD prevalence in Kyrgyzstan or neighbouring countries existed at the time of protocol development, we aimed for 600 participants based on a previous FRESH AIR study in Uganda [25] and on the BOLD (Burden of Lung Disease Initiative) study [26]. To this end, 50 households per setting were selected randomly through a multistage sampling approach. All permanent residents aged $\geqslant 18$ years who provided informed consent were included, because people highly exposed to HAP may develop COPD even before the age of 30 years [25]. Persons with a current respiratory infection or a contraindication for spirometry [27] were excluded. We obtained written, informed consent from all participants. All field researchers were trained on ethics and the informed consent procedure. The 
Research Ethics Committee of the National Centre for Cardiology and Internal Medicine (Bishkek, Kyrgyzstan) approved the study (protocol 9;2014/3/1).

\section{Demographics, COPD risk factors and respiratory symptoms}

A standardised questionnaire, composed of validated questionnaires and used in the FRESH AIR study in Uganda [25], was tailored to Kyrgyzstan (supplementary appendix S1). It was researcher-administered in the local language (Kyrgyz or Russian). It included questions on demographics, cooking and heating circumstances, COPD risk factors, and respiratory symptoms (tables 1 and 2).

\section{Household air pollution}

HAP was measured by the time-weighted average concentration of particulate matter with an aerodynamic diameter $<2.5 \mu \mathrm{m}(\mathrm{PM} 2.5)$ using a SidePak Personal Aerosol Monitor AM510 (TSI, Minneapolis, MN, USA). The cooks in the households were instructed to wear the device continuously on their body throughout the measurements. Measurements mostly started in the morning (median time 11:41 versus $11: 48 \mathrm{~h}$, respectively) and hence involved preparation of at least one warm meal. The median time of continuous measurement was $269 \mathrm{~min}$ in the lowlands and $284 \mathrm{~min}$ in the highlands (supplementary appendix S2). Of note, we initially aimed to measure 24-h indoor concentrations but this was not feasible due to the limited possibility to charge the battery of the device in the rural areas.

\section{COPD diagnosis}

A local team of medical doctors, trained by teachers from Imperial College London (London, UK), assessed lung function using spirometry (EasyOne; Medizintechnik, Zurich, Switzerland) following joint American Thoracic Society (ATS)/ERS guidelines [28]. We determined forced expiratory volume in $1 \mathrm{~s}$ (FEV1) and forced vital capacity (FVC) before and after bronchodilation with $400 \mu \mathrm{g}$ salbutamol using a spacer. COPD was defined as having a post-bronchodilator FEV1/FVC ratio $<70 \%$ [29] and severity was classified according to Global Initiative for Chronic Obstructive Lung Disease (GOLD) stages [30]. Subjects with complete reversibility were included as non-COPD in the analyses (an asthma diagnosis was

\begin{tabular}{|c|c|c|c|}
\hline & Lowlands & Highlands & $\mathrm{p}$-value \\
\hline Subjects & 193 & 199 & \\
\hline Male & 100 (51.8) & $87(43.7)$ & 0.109 \\
\hline Age years & $44.4 \pm 13.6$ & $50.0 \pm 16.3$ & $<0.001$ \\
\hline Height $\mathrm{cm}$ & $166.3 \pm 8.9$ & $161.1 \pm 9.6$ & $<0.001$ \\
\hline Weight kg & $71.7 \pm 14.7$ & $67.4 \pm 13.7$ & 0.002 \\
\hline $\mathrm{BMI} \mathrm{kg} \cdot \mathrm{m}^{-2}$ & $25.9 \pm 4.7$ & $26.0 \pm 5.6$ & 0.793 \\
\hline Higher education $^{\#}$ & $54(28.0)$ & $10(5.0)$ & $<0.001$ \\
\hline Profession & & & $<0.001$ \\
\hline Primary sector & $4(2.1)$ & $92(46.2)$ & \\
\hline Secondary sector & 23 (11.9) & $8(4.0)$ & \\
\hline Tertiary/quaternary sector & $90(46.6)$ & $13(6.5)$ & \\
\hline Housewife/husband & $31(16.1)$ & $37(18.6)$ & \\
\hline Other & 45 (23.3) & $49(24.6)$ & \\
\hline Smoking status & & & $<0.001$ \\
\hline Never-smoker & $110(57.0)$ & $140(74.1)$ & \\
\hline Ex-smoker & $12(6.2)$ & $14(7.4)$ & \\
\hline Current smoker & $71(36.8)$ & 35 (18.5) & \\
\hline Male & 58 (81.7) & 30 (85.7) & 0.604 \\
\hline Pack-years ${ }^{+}$ & $4.0(1.6-11.5)$ & $11.0(2.0-24.5)$ & 0.009 \\
\hline Tuberculosis (ever-diagnosed) $)^{\S}$ & $0(0.0)$ & $3(2.0)$ & 0.086 \\
\hline \multicolumn{4}{|l|}{ Solid fuel } \\
\hline Solid fuel use & $145(75.1)$ & $199(100.0)$ & $<0.001$ \\
\hline Nonsolid fuel use & $193(100.0)$ & $82(41.2)$ & $<0.001$ \\
\hline
\end{tabular}

Data are presented as $\mathrm{n}, \mathrm{n}(\%)$, mean \pm SD or median (interquartile range). BMI: body mass index. ${ }^{\#}$ : the highest level of completed education above secondary education; ${ }^{\text {?: }}$ two missing values in the lowlands and 14 in the highlands; ${ }^{+}$: two missing values in the lowlands and six in the highlands; ${ }^{\S}: 46$ missing values, all in the highlands. 


\begin{tabular}{|c|c|c|c|}
\hline & Lowlands & Highlands & p-value \\
\hline Subjects & 193 & 199 & \\
\hline Post-BD FVC \% pred & $92.0(84.0-104.0)$ & $100.0(92.0-110.0)$ & $<0.001$ \\
\hline Post-BD FEV $1 \%$ pred & $90.0(83.0-101.5)$ & $92.0(85.0-104.0)$ & 0.091 \\
\hline Post-BD FEV1/FVC \% & $82.0(75.0-96.0)$ & $76.0(65.0-97.0)$ & 0.003 \\
\hline COPD & $20(10.4)$ & $73(36.7)$ & $<0.001$ \\
\hline Male & $16(80.0)$ & $36(49.3)$ & $<0.001$ \\
\hline Moderate/severe (GOLD stage $\geqslant 2$ ) & $14(70.0)$ & $18(24.7)$ & $<0.001$ \\
\hline $\mathrm{SpO}_{2} \%$ & $95.0(95.0-96.0)$ & $93.0(92.0-95.0)$ & $<0.001$ \\
\hline Chronic cough/sputum $\#$ & $15(7.8)$ & $47(23.6)$ & $<0.001$ \\
\hline Severe breathlessness (MRC dyspnoea scale $\geqslant 4$ ) & $6(3.1)$ & $22(11.1)$ & 0.003 \\
\hline
\end{tabular}

Data are presented as $\mathrm{n}$, median (interquartile range) or $\mathrm{n}(\%)$. Missing outcomes for lung function were excluded from the study (figure 1); there were no other missing values. BD: bronchodilation; FVC: forced vital capacity; FEV1: forced expiratory volume in $1 \mathrm{~s}$; GOLD: Global Initiative for Chronic Obstructive Lung Disease; $\mathrm{SpO}_{2}$ : arterial oxygen saturation measured by pulse oximetry; MRC: Medical Research Council. \#: chronic is defined as having symptoms for $\geqslant 2$ months.

suspected). Arterial oxygen saturation $\left(\mathrm{SpO}_{2}\right)$ was measured by pulse oximetry (YX302 Fingertip Pulse Oximeter; Timago International Group, Bielsko-Biała, Poland).

\section{Data analysis}

SPSS version 23 (IBM, Armonk, NY, USA) was used for the analysis. Baseline characteristics, COPD prevalence and HAP exposure across altitudes were compared with independent t-tests for normally distributed continuous variables, Mann-Whitney U-tests for nonnormally distributed variables and Chi-squared or Fisher's exact tests for categorical variables. Univariable and forced-entry multivariable logistic regression models, adjusting for clustering within households by generalised estimating equation analyses [31], were used to assess the independent association between risk factors and COPD. HAP and other known COPD predictors (age, sex, education level and pack-years of smoking) [12] and altitude were included as predictors. Tuberculosis (ever-diagnosed) was not included in the model due to the limited number of cases. Working in the primary or secondary sector was included as a predictor due to the assumed higher occupational exposure compared with the tertiary/quaternary sector [32]. HAP was categorised into tertiles (supplementary appendix S4), given the nonnormal distribution with high values (in this high range we did not assume linearity of HAP with the logit of the outcome). Multicollinearity was not evident in the model. p-values $<0.05$ and odds ratios with a $95 \%$ confidence interval excluding 1 were considered statistically significant.

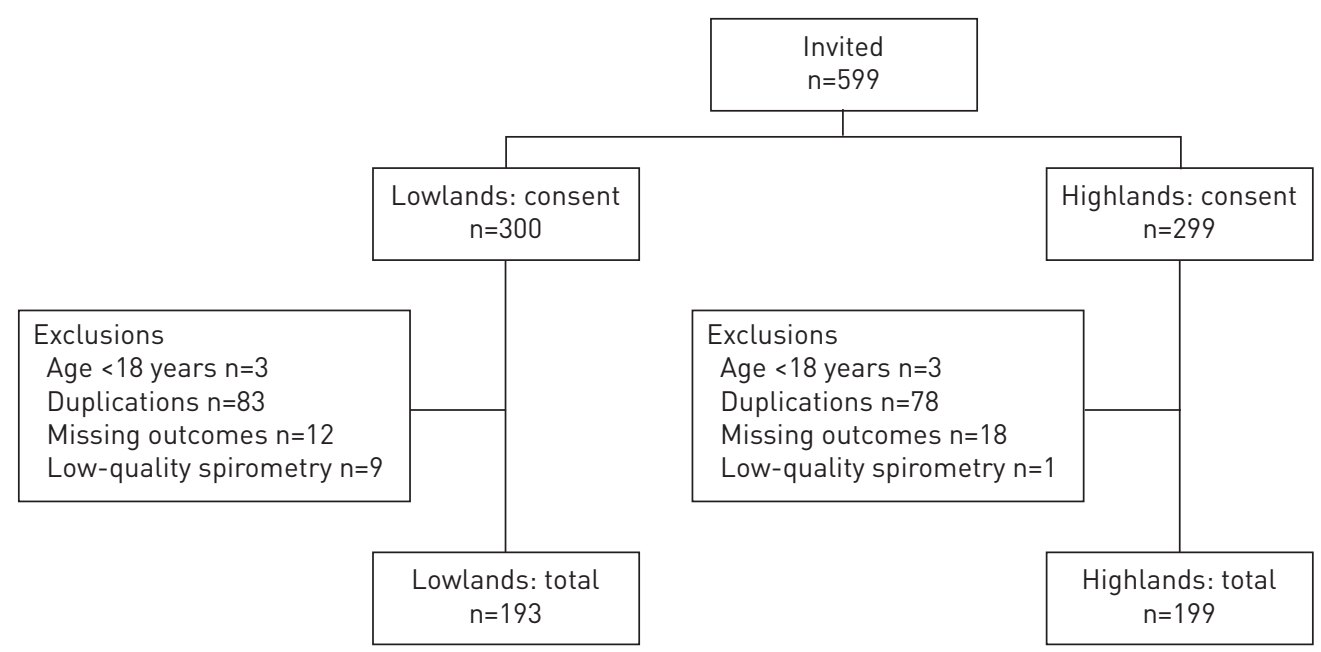

FIGURE 1 Flowchart of participants. 


\section{Handling of missing data}

Inadequate registration of several households and residents resulted in various duplications and uncertainty about these residents' PM2.5 measurements. To guarantee data quality and enable adjustment for a clustering effect within households, we discarded both these original and duplicate measurements from the analysis. Participants with missing PM2.5 and/or spirometry values were also excluded.

\section{Results}

Study population

All 599 invited individuals consented to participate in the study. After exclusion, 193 lowlanders and 199 highlanders from 41 households per setting remained for analysis (figure 1).

\section{Demographics and distribution of risk factors for COPD}

Highlanders were significantly older, and had lower height and weight (table 1). They had a lower level of education, and worked more often in the primary sector and less frequently in the secondary sector. The highland population consisted of significantly fewer smokers, although their pack-years were significantly higher. Highlanders were (nonsignificantly) more frequently ever-diagnosed with tuberculosis.

\section{Household air pollution across altitudes}

The time-weighted average PM2.5 concentration per household in the highlands was significantly higher than in the lowlands (median (interquartile range (IQR)) 290.0 (123.5-703.5) versus 72.0 (31.0167.0) $\mu \mathrm{g} \cdot \mathrm{m}^{-3} ; \mathrm{p}<0.001$ ) (figure 2). Furthermore, higher maximum PM2.5 concentrations were measured in the highlands (median (IQR) 5822.0 (2308.0-9152.5) versus $272.0(140.0-901.5) \mu \mathrm{g} \cdot \mathrm{m}^{-3}$; $\mathrm{p}<0.001$ ). In both areas these maxima were mostly reached at the beginning of the afternoon (median time 13:42:04 versus 13:05:40 h). Measurement duration is specified in supplementary appendix S2. The highlanders' cooking and heating circumstances were also more at risk of generating HAP. For example, highlanders significantly more often had higher-risk types of fuel use (e.g. dung versus gas), stoves (e.g. open fire versus improved cookstove), ventilation (e.g. eaves spaces versus open door), and cooking locations and durations. Details on these factors across the settings are provided in supplementary appendix S3.

\section{COPD prevalence across altitudes}

COPD was more prevalent in the highlands (36.7\% versus $10.4 \% ; \mathrm{p}<0.001)$, although severity was lower (GOLD stage $\geqslant 2: 24.7 \%$ versus $70.0 \%$; $\mathrm{p}<0.001$ ) (table 2 and figure 3 ). COPD was equally distributed among males and females in the highlands, while in the lowlands the vast majority of patients were male ( $49.3 \%$ versus $80.0 \%$; $\mathrm{p}<0.001$ ). COPD patients in the highlands were significantly less frequently smokers and were exposed to significantly higher PM2.5 levels compared with lowlanders (figure 4 and

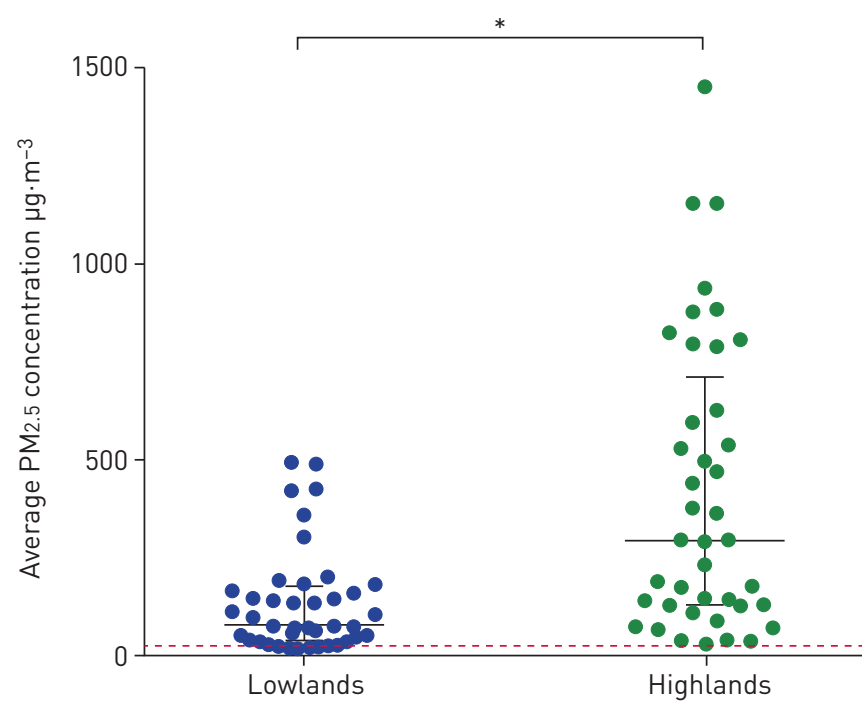

FIGURE 2 Levels of particulate matter with an aerodynamic diameter $<2.5 \mu \mathrm{m}$ (PM2.5) in the lowlands versus the highlands (measured as time-weighted average concentration per household). The median and interquartile range are indicated. The dashed line is the maximum average 24-h PM2.5 concentration of $25 \mu \mathrm{g} \cdot \mathrm{m}^{-3}$ as stated in the World Health Organization indoor air quality guideline [33]. Missing values were excluded from the study (figure 1). *: difference is statistically significant ( $p<0.001)$. 


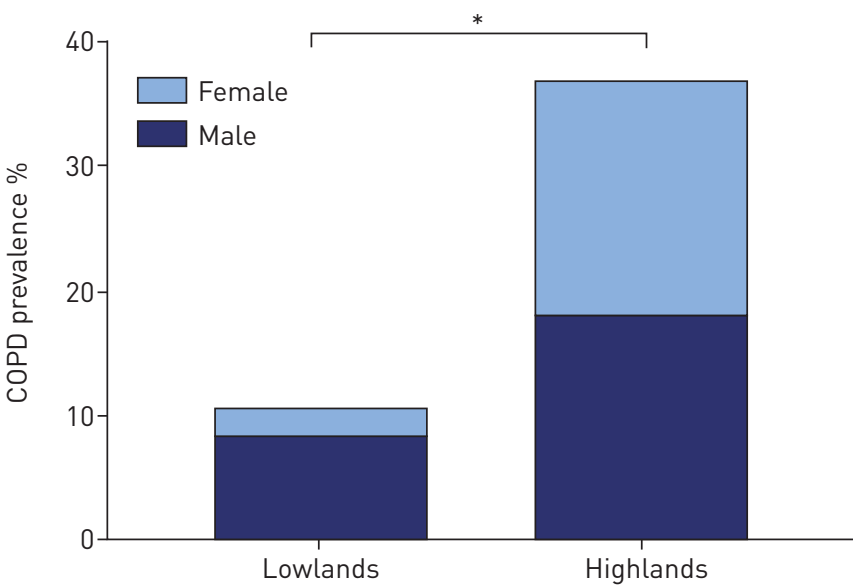

FIGURE 3 Chronic obstructive pulmonary disease (COPD) prevalence in the lowlands versus the highlands.

*: difference is statistically significant $(p<0.001)$.

supplementary appendix S4). Additionally, oxygen saturation was significantly lower among highlanders $\left(\mathrm{SpO}_{2}:\right.$ 93.0\% versus $\left.95.0 \% ; \mathrm{p}<0.001\right)$.

\section{Predictors for COPD}

Across the two altitudes, a total of 93 participants met COPD criteria. In the univariable logistic regression model, age, sex, pack-years, altitude and HAP were positively associated with COPD (figure 5 and supplementary appendix S4). In the multivariable logistic regression model, age, pack-years, altitude and the highest HAP exposure remained significantly positively associated with COPD. The decline in lung function along with an increase in PM2.5 exposure is depicted in supplementary appendix S4.

\section{Discussion}

In this study, we compared the prevalence of COPD and its risk factors between the highlands and lowlands in rural, low-resource settings in Kyrgyzstan, with a special focus on objectively measured HAP. Both COPD prevalence and HAP exposure (peak and average PM2.5 concentrations) were significantly higher among highlanders. In addition to living at high altitude and being exposed to high HAP concentrations, more pack-years and higher age were also independently associated with COPD (and more common in the highlands). Our results highlight the particular vulnerability of rural highlanders to COPD and confirm the increasingly acknowledged impact of HAP on respiratory health.

Overall COPD prevalence in both Kyrgyz settings was high considering our relatively low age inclusion criterion ( $\geqslant 18$ years). Our lowlands prevalence was similar to the global prevalence of $\sim 10 \%$, yet the prevalence observed in the highlands was more than 3-fold higher [26]. Our definition of COPD might have contributed to this high prevalence, especially among highlanders who were slightly older (a fixed FEV1/FVC ratio is known to overdiagnose COPD in the elderly [34]). Also, we used Caucasian reference

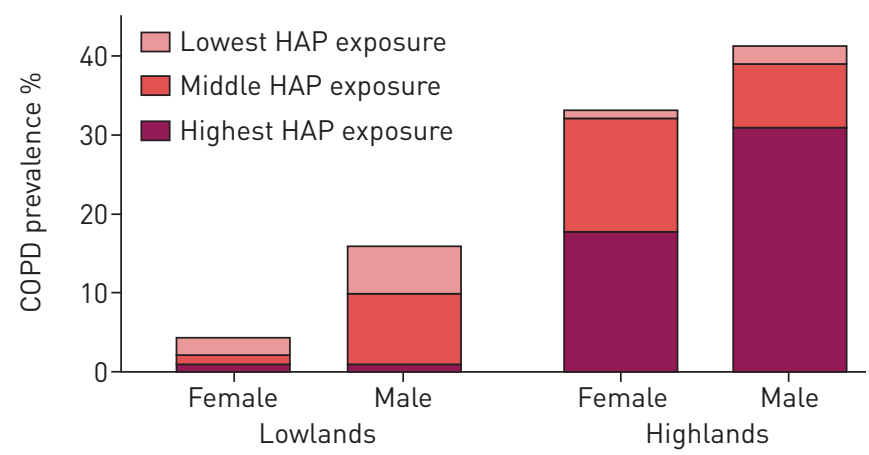

FIGURE 4 Chronic obstructive pulmonary disease (COPD) prevalence in the lowlands and highlands by sex and household air pollution (HAP) exposure. HAP exposure was categorised into tertiles, with time-weighted average concentrations of particulate matter with an aerodynamic diameter $<2.5 \mu \mathrm{m}$ of $\leqslant 72,>72-293$ and $>293 \mu \mathrm{g} \cdot \mathrm{m}^{-3}$. 

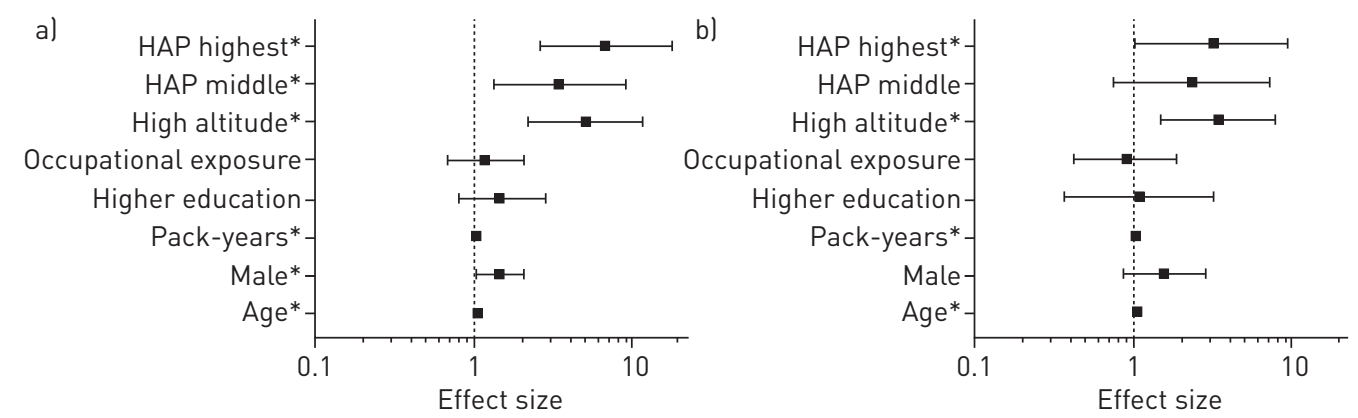

FIGURE 5 Potential predictors for chronic obstructive pulmonary disease (COPD): a) univariable analyses and b) multivariable analysis. HAP: household air pollution. COPD ( $n=93)$ versus no COPD ( $n=299$ ). Generalised estimating equation analyses adjusted for a clustering effect within households. Age per year increase. Higher education means the highest level of completed education is above secondary education. HAP exposure was categorised into tertiles, with time-weighted average concentrations of particulate matter with an aerodynamic diameter $<2.5 \mu \mathrm{m}$ of $\leqslant 72,>72-293$ and $>293 \mu \mathrm{g} \cdot \mathrm{m}^{-3}$. The lowest category is the reference category. Data are presented as OR $(95 \% \mathrm{CI})$. Note that with a COPD prevalence $>10 \%$, OR no longer approximates relative risk. ${ }^{*}$ : difference is statistically significant $(95 \% \mathrm{Cl}$ of the $\mathrm{OR}$ excluding $1 ; \mathrm{p}<0.05)$.

values (rural, high-altitude, Central Asian values do not exist), while a Kyrgyz study at middle altitude ( 1000-2000 m) reported larger FVC values among their population [35]. Larger physiological FVC values may result in smaller FEV1/FVC ratios and hence in overdiagnosis. A recent Kazakh population-based study found a prevalence of $6.7 \%$ [36]. The authors only studied a lowland, adult $(\geqslant 18$ years) population, used the same definition of COPD, but studied an urban rather than a rural setting. Given the lower use of solid fuels in urban settings, the latter factor may explain the lower COPD prevalence they observed.

In accordance with our hypothesis, along with a higher COPD prevalence in the highlands we observed significantly higher HAP exposures. It seems plausible to observe more HAP at higher altitude in rural areas, because more extreme climate conditions increase the need for (solid fuel-based) heating and for limiting ventilation. Furthermore, highlanders more commonly worked in the primary sector where dung is both cheaper and more easily available than clean fuels. Hence, particularly in the highlands, our HAP measurements dramatically exceeded the average $25 \mu \mathrm{g} \cdot \mathrm{m}^{-3}$ as stated in the WHO 24-h PM2.5 air quality guideline [33]. These measurements are consistent with PM2.5 concentrations in other households using predominantly biomass fuels [13]. Our findings support the assumption that HAP plays a substantial role in explaining a high COPD prevalence at high altitude. This assumption is furthermore consistent with the relatively more (nonsmoking) female patients in our highland population, like in other settings where women are highly exposed to biomass fuel smoke [13-15, 17, 25, 37]. Although the pathogenic mechanisms of the harm of HAP are not yet fully uncovered, HAP is considered to alter the innate immune response, enhance pulmonary and systemic inflammation, and promote an oxidative stress state. This may cause macromolecular cell damage, including DNA changes [38]. Nevertheless, BOLD results remind us to remain alert for causes other than HAP for airflow obstruction [39].

In addition to the strong and significant relation between COPD and PM2.5 concentrations, altitude itself was another independent predictor in the multivariable analysis, also after adjusting for age and pack-years. Altitude might directly impact COPD prevalence as it is associated with an increase in both airways and total lung capacities. However, evidence remains inconclusive if this increase is proportional (hence, if and how FEV1/FVC ratios are affected [40-42]). Alternatively, altitude could relate to COPD prevalence indirectly via (unmeasured) factors. These could be a more frequent history of pneumonia/ tuberculosis, lower level of physical activity [43, 44], higher occupational exposure [32] or more (childhood) poverty/lower sociodemographic development (as again a mediator for other factors) [2, 45].

Remarkably, prevalence of moderate/severe COPD (GOLD stage $\geqslant 2$ ) appeared to be lower in the highlands. This could be due to down-migration of the severely ill for higher ambient oxygen levels [46], for better access to advanced healthcare in the Kyrgyz capital or to a potential relation between severity and solid fuel smoke versus tobacco smoke [37]. The lower highland oxygen saturation levels we observed seem plausible due to lower ambient oxygen levels in the highlands. Furthermore, a double exposure of tobacco and biomass fuel smoke among COPD patients is associated with lower oxygen saturation levels [47]. However, although the number of COPD patients with a double exposure was significantly higher in the highlands, the total number of participants with a double exposure was the same.

As mentioned earlier, the seven other studies covering COPD prevalence across altitudes reported conflicting findings [6-12]. We argue that these conflicting results can be partly explained by differences 
in HAP exposure, which these studies either did not measure, or measured by proxy, or did not adjust for in the analyses. HAP exposure, associated COPD prevalence and the difference across altitudes may have differed substantially between the study settings. The settings were almost exclusively urban, sometimes in a high-income country, meaning more extreme high-altitude climates could be less influential on HAP exposure due to better access to clean fuels [14]. Furthermore, ethnicity often differed between the highand low-altitude settings within a study, hampering proper comparison of lung functions.

We corrected for objectively measured HAP in our analyses, compared consistently between rural, low-resource settings and between people of the same ethnicity. Altitude remained an independent predictor for COPD. The conflicting results between all present studies, performed in different settings, may indicate COPD prevalence might not be attributable to altitude itself. Instead, it could be mainly mediated through (unmeasured) factors expressed by altitude, with HAP being one of them.

A major strength of this study is the objective, personally monitored PM2.5 exposure combined with spirometry according to ATS/ERS guidelines [28], other than self-reported presence of risk factors or symptoms. This method precisely answers a recent call for objective measurements on the association between HAP and COPD [20]. This study also benefited from a high participation rate. Participants indicated they were eager to receive extra medical attention as access to healthcare is generally limited in these rural areas. This high rate impedes selection bias and enhances generalisability to other settings. Lastly, we present one of the first spirometry-based COPD prevalence studies in Central Asia [2].

Some limitations must also be noted. Due to organisational difficulties during data collection, we had to exclude various participant and household measurements in both settings. Differences between the two settings were, however, still statistically significant, and we were not able to identify any differences between excluded and included participants. Also, due to the limited battery and charging capacity in the rural areas, we could not perform 24-h PM2.5 measurements. We therefore measured each household for $\sim 4 \mathrm{~h}$ and at the same hour during the day, including the preparation of at least one warm meal. Hence, 24-h PM2.5 concentrations are likely to be lower as those contain a longer period without meal preparations. Lastly, given that the data in the lowlands were collected in a relatively colder period of the year, with a relatively higher need for (solid fuel-based) heating and limiting ventilation, differences in HAP and respiratory symptoms might be underestimated.

In conclusion, we observed a substantially higher COPD prevalence in high-altitude areas compared with low-altitude areas in rural Kyrgyzstan. HAP exposure was high in both settings, but particularly in the highlands. There was a strong, significant relation between COPD and HAP that remained significant when adjusted for other risk factors. Although generalisability to other rural, low-resource highlands remains to be assessed, our results call for an increased focus on COPD awareness, diagnosis and treatment. Moreover, policy makers, healthcare workers and highlanders in these settings should focus on prevention by increasing awareness on HAP and by reducing it (e.g. by the implementation of clean cooking stoves). This could not only benefit chronic lung health, but also target other PM2.5-related diseases such as ischaemic heart disease, cerebrovascular disease, lung cancer, low birthweight and pre-term birth, type 2 diabetes, and lower respiratory infections [16].

Acknowledgements: This study was based on a previous FRESH AIR study in Uganda. We thank Frederik van Gemert (University Medical Center Groningen, Groningen, The Netherlands) and co-authors for sharing their protocol and questionnaire. We thank Beraki Ghezai (Lunger i Praksis, Oslo, Norway) for his important role in introducing the programme in Kyrgyzstan, educating the researchers and translating the protocol to Russian. We also thank Ron Wolterbeek (retired) and Nan van Geloven, statisticians at Leiden University Medical Center (LUMC; Leiden, The Netherlands), for statistical input. We thank Charlotte Poot (LUMC) for her support in designing the figures. We thank the International Primary Care Respiratory Group for securing the grant from the European Lung Foundation. Lastly, we thank all participants. This paper was presented as an oral presentation at the ERS International Congress, Paris, France, September 15-19, 2018 (OA288).

Author contributions: T. Sooronbaev and A. Tabyshova adapted the FRESH AIR study to the Kyrgyz settings and coordinated data collection. E.A. Brakema, A. Tabyshova, M.J. Kasteleyn and E. Molendijk analysed and interpreted data. E.A. Brakema wrote the first draft of this report. All authors provided comments and E.A. Brakema revised the report. All authors gave input to the final version. All authors had full access to all the data. E.A. Brakema and N.H. Chavannes had the final responsibility for the decision to submit the study for publication.

Conflict of interest: None declared.

Support statement: This study was funded by Healthy Lungs for Life from the European Lung Foundation. The funders had no role in study design, data collection, analysis, interpretation or writing of the report.

\section{References}

1 Institute for Health Metrics and Evaluation. GBD compare data visualization. 2016. http://vizhub.healthdata.org/ gbd-compare Date last accessed: April 4, 2018. 
2 GBD Chronic Respiratory Disease Collaborators. Global, regional, and national deaths, prevalence, disability-adjusted life years, and years lived with disability for chronic obstructive pulmonary disease and asthma, 1990-2015: a systematic analysis for the Global Burden of Disease Study 2015. Lancet Respir Med 2017; 5: 691-706.

3 World Health Organization. Chronic obstructive pulmonary disease (COPD). 2016. www.who.int/mediacentre/ factsheets/fs315/en Date last accessed: April 4, 2018.

4 Khakban A, Sin DD, FitzGerald JM, et al. Ten-year trends in direct costs of COPD: a population-based study. Chest 2015; 148: 640-646.

5 Cohen JE, Small C. Hypsographic demography: the distribution of human population by altitude. Proc Natl Acad Sci USA 1998; 95: 14009-14014.

6 Caballero A, Torres-Duque CA, Jaramillo C, et al. Prevalence of COPD in five Colombian cities situated at low, medium, and high altitude (PREPOCOL study). Chest 2008; 133: 343-349.

7 Menezes AM, Perez-Padilla R, Jardim JR, et al. Chronic obstructive pulmonary disease in five Latin American cities (the PLATINO study): a prevalence study. Lancet 2005; 366: 1875-1881.

8 Laniado-Laborin R, Rendon A, Batiz F, et al. High altitude and chronic obstructive pulmonary disease prevalence: a casual or causal correlation? Arch Bronconeumol 2012; 48: 156-160.

9 McDaniel JT. Prevalence of chronic obstructive pulmonary disease: county-level risk factors based on the Social Ecological Model. Perspect Public Health 2018; 138: 200-208.

10 Miele CH, Grigsby MR, Siddharthan T, et al. Environmental exposures and systemic hypertension are risk factors for decline in lung function. Thorax 2018; 73: 1120-1127.

11 Aaron SD, Gershon AS, Gao Y, et al. Influence of country-level differences on COPD prevalence. Int J Chron Obstruct Pulmon Dis 2016; 11: 2305-2313.

12 Horner A, Soriano JB, Puhan MA, et al. Altitude and COPD prevalence: analysis of the PREPOCOL-PLATINO-BOLD-EPI-SCAN study. Respir Res 2017; 18: 162.

13 Gordon SB, Bruce NG, Grigg J, et al. Respiratory risks from household air pollution in low and middle income countries. Lancet Respir Med 2014; 2: 823-860.

14 World Health Organization. Fuel for Life: Household Energy and Health. Geneva, WHO, 2006.

15 Salvi SS, Barnes PJ. Chronic obstructive pulmonary disease in non-smokers. Lancet 2009; 374: 733-743.

16 Cohen AJ, Brauer M, Burnett R, et al. Estimates and 25-year trends of the global burden of disease attributable to ambient air pollution: an analysis of data from the Global Burden of Diseases Study 2015. Lancet 2017; 389: 1907-1918.

17 Siddharthan T, Grigsby MR, Goodman D, et al. Association between household air pollution exposure and chronic obstructive pulmonary disease outcomes in 13 low- and middle-income country settings. Am J Respir Crit Care Med 2018; 197: 611-620.

18 Magitta NF, Walker RW, Apte KK, et al. Prevalence, risk factors and clinical correlates of COPD in a rural setting in Tanzania. Eur Respir J 2018; 51: 1700182.

19 GBD Risk Factors Collaborators. Global, regional, and national comparative risk assessment of 79 behavioural, environmental and occupational, and metabolic risks or clusters of risks, 1990-2015: a systematic analysis for the Global Burden of Disease Study 2015. Lancet 2016; 388: 1659-1724.

20 Balmes JR, Eisen EA. Household air pollution and chronic obstructive pulmonary disease. "A riddle, wrapped in a mystery, inside an enigma". Am J Respir Crit Care Med 2018; 197: 547-549.

21 World Bank. Country profile: Kyrgyz Republic. 2016. http://databank.worldbank.org/data/Views/Reports/ ReportWidgetCustom.aspx?Report_Name=CountryProfile\&Id=b450fd57\&tbar=y\&dd=y\&inf=n\&zm=n\&country=KGZ Date last accessed: June 16, 2018.

22 Gibson J, Loddenkemper R, Sibille Y, et al., eds. The Burden of Lung Disease. In: European Lung White Book. Sheffield, European Respiratory Society, 2013.

23 National Statistical Committee of the Kyrgyz Republic. Population. 2018. www.stat.kg/en/opendata/category/4 Date last accessed: November 18, 2018.

24 Cragg L, Williams S, Chavannes NH. FRESH AIR: an implementation research project funded through Horizon 2020 exploring the prevention, diagnosis and treatment of chronic respiratory diseases in low-resource settings. NPJ Prim Care Respir Med 2016; 26: 16035.

25 van Gemert F, Kirenga B, Chavannes N, et al. Prevalence of chronic obstructive pulmonary disease and associated risk factors in Uganda (FRESH AIR Uganda): a prospective cross-sectional observational study. Lancet Glob Health 2015; 3: e44-e51.

26 Buist AS, McBurnie MA, Vollmer WM, et al. International variation in the prevalence of COPD (the BOLD Study): a population-based prevalence study. Lancet 2007; 370: 741-750.

27 Cooper BG. An update on contraindications for lung function testing. Thorax 2011; 66: 714-723.

28 Miller MR, Hankinson J, Brusasco V, et al. Standardisation of spirometry. Eur Respir J 2005; 26: 319-338.

29 Fabbri LM, Hurd SS. Global Strategy for the Diagnosis, Management and Prevention of COPD: 2003 update. Eur Respir J 2003; 22: 1-2.

30 Global Initiative for Chronic Obstructive Lung Disease. Global Strategy for the Diagnosis, Management, and Prevention of Chronic Obstructive Pulmonary Disease. 2017. http://goldcopd.org/gold-2017-global-strategydiagnosis-management-prevention-copd Date last accessed: November 18, 2018.

31 Zeger SL, Liang KY. Longitudinal data analysis for discrete and continuous outcomes. Biometrics 1986; 42: 121-130.

32 World Health Organization. Preventing Noncommunicable Diseases (NCDs) by Reducing Environmental Risk Factors. Geneva, WHO, 2017.

33 World Health Organization. WHO Indoor Air Quality Guidelines: Household Fuel Combustion. Geneva, WHO, 2014.

34 Hnizdo E, Glindmeyer HW, Petsonk EL, et al. Case definitions for chronic obstructive pulmonary disease. COPD 2006; 3: 95-100.

35 Vinnikov D, Blanc PD, Brimkulov N. Spirometry reference values for a Kyrgyz population. Clin Respir J 2018; 12 : 826-828. 
36 Nugmanova D, Feshchenko Y, Iashyna L, et al. The prevalence, burden and risk factors associated with chronic obstructive pulmonary disease in Commonwealth of Independent States (Ukraine, Kazakhstan and Azerbaijan): results of the CORE study. BMC Pulm Med 2018; 18: 26.

37 Ramirez-Venegas A, Sansores RH, Perez-Padilla R, et al. Survival of patients with chronic obstructive pulmonary disease due to biomass smoke and tobacco. Am J Respir Crit Care Med 2006; 173: 393-397.

38 Olloquequi J, Silva OR. Biomass smoke as a risk factor for chronic obstructive pulmonary disease: effects on innate immunity. Innate Immun 2016; 22: 373-381.

39 Amaral AFS, Patel J, Kato BS, et al. Airflow obstruction and use of solid fuels for cooking or heating: BOLD results. Am J Respir Crit Care Med 2017; 197: 595-610.

40 Havryk AP, Gilbert M, Burgess KR. Spirometry values in Himalayan high altitude residents (Sherpas). Respir Physiol Neurobiol 2002; 132: 223-232.

41 Wood S, Norboo T, Lilly M, et al. Cardiopulmonary function in high altitude residents of Ladakh. High Alt Med Biol 2003; 4: 445-454.

42 Rupert JL, Hochachka PW. The evidence for hereditary factors contributing to high altitude adaptation in Andean natives: a review. High Alt Med Biol 2001; 2: 235-256.

43 Lee $\mathrm{CH}$, Lee MC, Lin HH, et al. Pulmonary tuberculosis and delay in anti-tuberculous treatment are important risk factors for chronic obstructive pulmonary disease. PLoS One 2012; 7: e37978.

44 Garcia-Aymerich J, Lange P, Benet $\mathrm{M}$, et al. Regular physical activity modifies smoking-related lung function decline and reduces risk of chronic obstructive pulmonary disease: a population-based cohort study. Am J Respir Crit Care Med 2007; 175: 458-463.

45 Townend J, Minelli C, Mortimer K, et al. The association between chronic airflow obstruction and poverty in 12 sites of the multinational BOLD study. Eur Respir J 2017; 49: 1601880.

46 Regensteiner JG, Moore LG. Migration of the elderly from high altitudes in Colorado. JAMA 1985; 253: 3124-3128.

47 Olloquequi J, Jaime S, Parra V, et al. Comparative analysis of COPD associated with tobacco smoking, biomass smoke exposure or both. Respir Res 2018; 19: 13. 